\title{
Testing Rheological Properties of Alkali-Activated Cement Using Non-Traditional Test Methods
}

\author{
Gediminas Kastiukas $^{1 *}$, Xiangming Zhou ${ }^{1}$, and Woon Y. Yang ${ }^{1}$ \\ ${ }^{1}$ Department of Civil and Environmental Engineering, Brunel University, London, United Kingdom
}

\begin{abstract}
In this paper, the rheological parameters of alkaline activated cement (AAC) paste tailored for continuous extrusion using the non-traditional test method of the direct shear box test are presented. Characteristic changes of the material's rheological behaviour are estimated in response to variations in the water-to-solid ratio and addition of a cellulosic rheology aid. The results indicate that the increase of the water-to-solid ratio from 0.18 to 0.19 does not impact the internal angle of friction $(\varphi)$ but does, however, reduce the shear yield strength, or cohesion $(c)$, by $85 \%$. For mixes containing cellulosic rheology aid, the internal angle of friction shows no significant change also. However, the shear yield strength for the mix with a water-to-solid ratio of 0.18 is $51 \%$ higher. The shear yield strength increases by a further $28 \%$ for the mix with a water-to-solid ratio of 0.19 . These results indicate that the cellulosic rheology aid does not necessarily increase the "flow-like" characteristics of the mix which provides it plasticity and the ability to move through the extruder. It does however significantly improve its 'green strength', even at an increased water-to-solid ratio of 0.19 , which would ultimately assist with lowering the binder density.
\end{abstract}

\section{Introduction}

As an economical, energy efficient, and continuous material processing technology, extrusion is a widelyused industrial process in plastic, food, ceramic, hightemperature metal alloy, pharmaceuticals and clay industries. In an effort to harness the high potential of the extrusion processing technology, the concrete industry has introduced this continuous manufacturing process for cement-based building products like flat and corrugated sheets, solid façade panels, honeycomb panels, window/door frames, door panels, roof tiles and downpipes [1]-[5]. Also, significant EU research funding has been granted to promote the development of extrusion-based cementitious materials, with particular emphasis on tailoring the extrusion process and mix formulations [6].

A successful extrusion process largely depends on the material rheological properties, as well as on the extrusion system itself [7]. For the material to be suitable for extrusion, it should satisfy some specific material properties. For example, components making up the composite should exhibit homogeneous dispersion; be soft enough for extrusion through the die continuously with reasonably low energy consumption; have sufficient 'green strength' to maintain its shape upon exiting the die, and have an appropriate deformability rate so that a satisfactory production rate could be achieved. Rheological properties have been traditionally measured using test methods developed primarily for highly flowable mixtures like traditional cement pastes, mortars and concrete which usually have a much higher water-tosolid ratio. However, the rheological behaviour of semisolid fresh cementitious materials is largely different from that of traditional cement paste, mortar, concrete [7]. So far, limited quantitative data exist for describing the rheological properties of the semi-solid fresh cementitious materials for extrusion, mainly due to the complexity of the material rheological properties and also a lack of appropriate rheology test methods [8]. It is almost impossible to derive all the elastic, viscous, plastic and cohesive rheological properties of such semisolid fresh cement-based materials from one single rheology test method. Zhou et al. [7] summarised the 'non-traditional' rheology test methods for fibrereinforced semi-solid fresh cement-based materials for extrusion. However, few studies have reported on directly measuring shear yield strength for extrusion of semi-solid fresh cementitious materials, especially of the alkali-activated type. Alkali-activated cementitious materials are based on a reactive solid substance that hardens under the influence of an alkaline activator and can be described as three-dimensionally networked amorphous to semi-crystalline alumino-silicate materials. These materials have the potential to be made from a variety of industrial waste sources, many of which have remained largely unexplored.

The direct shear box test is widely used for testing shear parameters of geomaterials because of its simplicity in operation and suitability for a wide range of geomaterials [9]. In this study, this technique was employed to

"Corresponding author: gediminas.kastiukas@brunel.ac.uk 
investigate the rheology of semi-solid fresh alkaliactivated materials tailored for extrusion. To obtain the relationship between the measured shear yield strength and normal stress (i.e. consolidation pressure), a range of fresh tungsten mining waste/waste glass alkali-activated binders were tested under varying consolidation pressures. It is also vital to figure out the effects of individual material components on the overall rheological properties of the binders. In this study, the effects of water-to-solid ratio and cellulosic rheology aid dosage were investigated to help tailor material formulation design and achieve successive manufacturing.

\section{Materials and Methods}

\subsection{Materials}

Raw materials for preparing the semi-solid mixtures included tungsten mining waste (TMW), milled waste glass (WG). The chemical compositions of the raw powders, as determined by WD-XRF, are presented in Table 1. Sodium hydroxide (SH), sodium silicate (SS) were used for the preparation of the alkali activator. Finally, hydroxyethyl methyl cellulose (WALOCELTM VP-M-7701, Dow Chemicals, EU) was used to improve the rheology of the fresh alkali-activated binder by enabling the mixtures to reach dough-like plasticity and enhance their water retention capacity.

In this study, four formulations (Table 2) were prepared and subjected to the direct shear test. The formulations were named based on the water-to-solid ratio and presence of rheology aid. For example, mix 0.19-P had a water-to-solid ratio of 0.19 and was plain (P) i.e. did not contain rheology aid.

Table 1. Chemical composition of TMW and WG

\begin{tabular}{ccc}
\hline Component & TMW Result (\%) & WG Result (\%) \\
\hline $\mathrm{Na} 2 \mathrm{O}$ & 0.51 & 12.44 \\
$\mathrm{MgO}$ & 2.16 & 1.76 \\
$\mathrm{~A} 2 \mathrm{O} 3$ & 14.89 & 2.12 \\
$\mathrm{SiO} 2$ & 49.17 & 68.71 \\
$\mathrm{SO} 3$ & 8.98 & 0.33 \\
$\mathrm{~K} 2 \mathrm{O}$ & 2.92 & 0.77 \\
$\mathrm{Fe} 2 \mathrm{O} 3$ & 13.69 & 1.48 \\
$\mathrm{CaO}$ & 0.58 & 10.04 \\
$\mathrm{P} 2 \mathrm{O} 5$ & 0.32 & 0.00 \\
$\mathrm{TiO} 2$ & 0.5 & 0.00 \\
$\mathrm{ZnO}$ & 1.25 & 0.00 \\
$\mathrm{CuO}$ & 0.32 & 0.00 \\
$\mathrm{As} 2 \mathrm{O} 3$ & 4.26 & 0.00 \\
\hline
\end{tabular}

Table 2. AAC paste mixture formulations

\begin{tabular}{ccccc}
\hline & \multicolumn{4}{c}{ Mix } \\
& $0.18-\mathrm{P}$ & $0.19-\mathrm{P}$ & $0.18-\mathrm{RA}$ & $0.19-\mathrm{RA}$ \\
\hline TMW (g) & 274.0 & 274.0 & 274.0 & 274.0 \\
WG (g) & 182.0 & 182.0 & 182.0 & 182.0 \\
SH (g) & 26.0 & 26.0 & 26.0 & 26.0 \\
SS (g) & 75.0 & 75.0 & 75.0 & 75.0 \\
Water (g) & 27.4 & 32.0 & 27.40 & 32.0 \\
Rheology aid (g) & 0 & 0 & 4.57 & 4.57 \\
\hline
\end{tabular}

In all formulations, the mass ratio of TMW to milled WG was kept constant at 1.8. The SS/SH mass ratio was also kept constant at 2.9. The precursor-to-activator mass ratio for all formulation was 4.5. The only variables were the dosage of water and the addition of rheology aid. Additional water was added to the mixture as $6 \%$ and $7 \%$ by weight of solid materials (TMWM and milled waste glass). The rheology aid was added as $1 \%$ by weight of solid materials. The water-to-solid ratio investigated in this study is defined as the ratio of the total mass of water (water in the activating solution and additional water) to the total mass of binder solids (TMW, milled WG and solids in the activating solution). The water-to-solid ratio for mix 0.18-P, 0.19-P, 0.18-RA and $0.19-\mathrm{RA}$ were $0.18,0.19,0.18$ and 0.19 , respectively.

\subsection{Methods}

\subsubsection{Shear Box Test}

A lever loading direct shear box device (SHEARTEST VJT2760) was used to test the AAC pastes. This device utilises weights and a lever loading piston for applying a vertical load to the sample while mechanical dial indicators measure the vertical and shear displacements. The shear stress is obtained by converting the measurement of the horizontal dial gauge attached to the load cell. The shear box specimen is sandwiched between two perforated plates and two porous plates followed by a loading pad on top. In the shear box test, the sample is consolidated first under a normal load, i.e., primary consolidation. Upon the completion of the primary consolidation, the sample is sheared horizontally along its mid-height plane (between the two halves of the shear box). The shearing resistance offered by the material sample in the shear box is recorded during the test. Yielding occurs when the shearing resistance reaches the maximum value which is usually represented by a plateau in the measured shear stress vs shear displacement curve obtained from experiment. The horizontal shear load $\mathrm{P}$ is obtained by multiplying the reading of the dial gauge by a calibration coefficient of ring stiffness ratio $(0.0021625 \mathrm{kN} / \mathrm{Div})$. The true shear stress $\pi$ on the shear surface of a prismatic sample is calculated as: 


$$
\pi=\frac{\mathrm{P}}{\mathrm{A}_{\mathrm{c}}}
$$

where $A_{c}$ is the corrected area. The area is obtained by multiplying the width by length. The length of the shear box is fixed at $60 \mathrm{~mm}$. However, the width of the shear box changes due to horizontal displacement during the test. The width of the shear box is calculated by subtracting its initial width of $60 \mathrm{~mm}$ by the displacement $(\mathrm{mm})$. Therefore, the area cannot be assumed to be constant throughout the whole test.

The normal stress $\sigma_{\mathrm{n}}$, applied to the specimen can be obtained by the following equation,

$$
\sigma_{n}=\frac{\mathrm{F}}{\mathrm{A}}
$$

where $\mathrm{F}$ is, the force being applied vertically on the sample and $\mathrm{A}$ is the area of the specimen which is constant throughout the test.

With the known shear strength and vertical normal stress, the Mohr-Coulomb model is used to study the relation between shear yield strength and vertical normal stress for materials exhibiting both friction and cohesion. The Mohr-Coulomb model is expressed as:

$$
\tau_{\mathrm{f}}=\mathrm{c}+\sigma_{\mathrm{n}} \tan \varphi
$$

where $\mathrm{c}$ is the cohesion and $\varphi$ is the angle of internal friction. The cohesion is the shear strength of the sample under zero normal stress. Cohesion and the angle of shearing resistance can be obtained by plotting a linear graph of maximum shear stress against normal stress. By performing the test under 3 different normal loads, the best-fitted line can be plotted. The y-intercept of the fitted line gives a value for cohesion, and the angle of the fitted line gives the friction angle.

Each formulation was tested under a nominal stress $\left(\sigma_{n}\right)$ of $65.7,124.8$, and $174.6 \mathrm{kPa}$, with three samples tested under each value of normal stress. The shear displacement rate was kept constant at $1 \mathrm{~mm} / \mathrm{min}$ for all formulations. In total, 12 samples were prepared and tested using the direct shear box. The duration of a single shear box test was $20 \pm 1$ minutes after sample mixing was complete, which was consistent with the fact that the freshly prepared cementitious materials are expected to be extruded out of die to form the desired products 20 minutes after mixing.

\subsubsection{Alkali-Activated Binder Preparation}

The fresh mixtures were all prepared with a commercial bench-top mixer (Kenwood Major KM020). First, all powder components, in this case, TMW, WG, rheology aid (if used in the formulation), were blended in the mixer for 5 minutes at the speed of $60 \mathrm{rpm}$. Then, the liquid components, in this case, alkali activator and deionised water were added to the mixture. The slurry was then blended for another 2.5 minutes at the speed of $60 \mathrm{rpm}$. Then, a higher speed (120 rpm) was adjusted, and the slurry was blended for a further 7.5 minutes until a semi-solid dough-like material was formed.

\section{Results and Discussion}

A typical Mohr-Coulomb failure envelope obtained for mix 0.18-P is shown in Fig. 1. These data were subsequently used to determine the angle of internal friction and cohesion. The estimates of the friction angle and cohesion developed from the data for all four mixes are summarised in Table 3. mix 0.18-P and mix 0.19-P were made with water-to-solid ratios of 0.18 and 0.19 , respectively. The two mixes were evaluated to maintain very similar angles of internal friction, namely $38.8^{\circ}$ and $37.7^{\circ}$, respectively. However, the cohesion for mix $0.18 \mathrm{P}$ was $6.25 \mathrm{kPa}$, while for mix $0.19-\mathrm{P}$, it was $0.94 \mathrm{kPa}$. The latter results reveal that the increase of the water-to-solid ratio has a negligible impact on the angle of internal friction, however, imparts an $85 \%$ reduction in the cohesion of the AAC paste. Although the cohesion and shear yield strength is significantly higher when the AAC paste has a lower water-to-solid ratio, the stiffness of the AAC paste is much greater, making it less favourable for extrusion purposes.

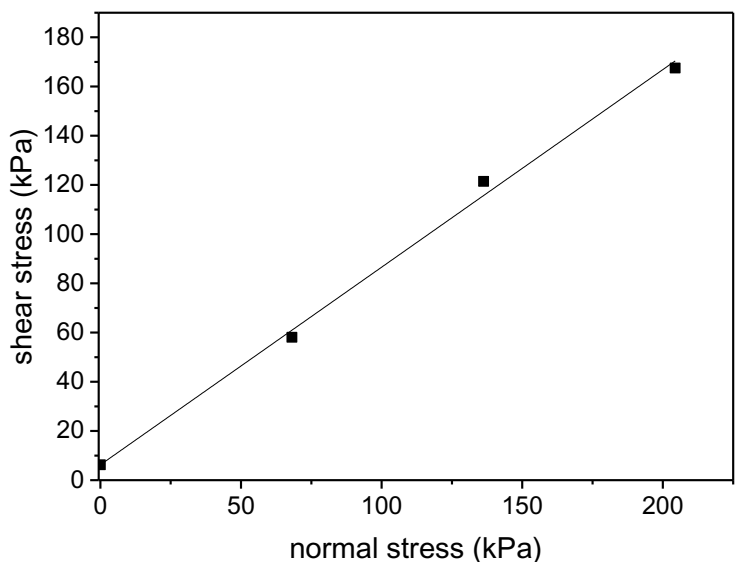

Fig. 1. Maximum shear stress vs normal stress for mix 0.18-P

Mix 0.18-RA was made with a water-to-solid ratio of 0.18 but with the addition of $1 \%$ rheology aid by weight of solids. The angle of internal friction of 0.18 -RA was $38.6^{\circ}$ and again, very much like the formulations without rheology aid (mix 0.18-P and mix 0.19-P). Nonetheless, the cohesion for mix 0.18 -RA was $12.75 \mathrm{kPa}$; more than double the cohesion value obtained by the equivalent 
mix without rheology aid (mix 0.18-P). Finally, when a water-to-solid ratio of 0.19 was made in combination with the rheology aid (mix 0.19-RA), the angle of internal friction was measured to moderately reduce by $5 \%$ while the cohesion increased by $28 \%$. This reveals that for an AAC paste containing a cellulosic rheology aid, an incremental increase in the water-to-solid ratio significantly increases the materials cohesion. The opposite trend applies for AAC paste without rheology aid (mix 0.18-P and 0.19-P), where an increase of waterto-solid ratio leads to lower cohesion, i.e. shear yield strength.

Table 3. Summary of rheological parameters for all mixes under direct shear box test

\begin{tabular}{ccccc}
\hline & $0.18-\mathrm{P}$ & $0.19-\mathrm{P}$ & $0.18-\mathrm{RA}$ & $0.19-\mathrm{RA}$ \\
\hline $\begin{array}{c}\text { Water-to-solid } \\
\text { ratio }\end{array}$ & 0.18 & 0.19 & 0.18 & 0.19 \\
$\begin{array}{c}\text { Rheology aid } \\
(\%)\end{array}$ & 0 & 0 & 1 & 1 \\
$\begin{array}{c}\text { angle of internal } \\
\text { friction }\left({ }^{\circ}\right)\end{array}$ & 38.8 & 37.7 & 38.6 & 36.7 \\
Cohesion $(\mathrm{kPa})$ & 6.25 & 0.94 & 12.75 & 17.62 \\
\hline
\end{tabular}

Fig. 2 shows the vertical displacement against consolidation time for all four mix formulations. The consolidation displacement of mix 0.18-RA was evaluated to be less than mix 0.19-RA. The consolidation displacements of mix formulations without rheology aid (mix 0.18-P and mix 0.19-P) are higher than the mix formulations with rheology aid (mix 0.18RA and 0.19-RA). This confirms that the presence of rheology aid makes the AAC paste stiffer, indicating more force would be needed for successful extrusion.

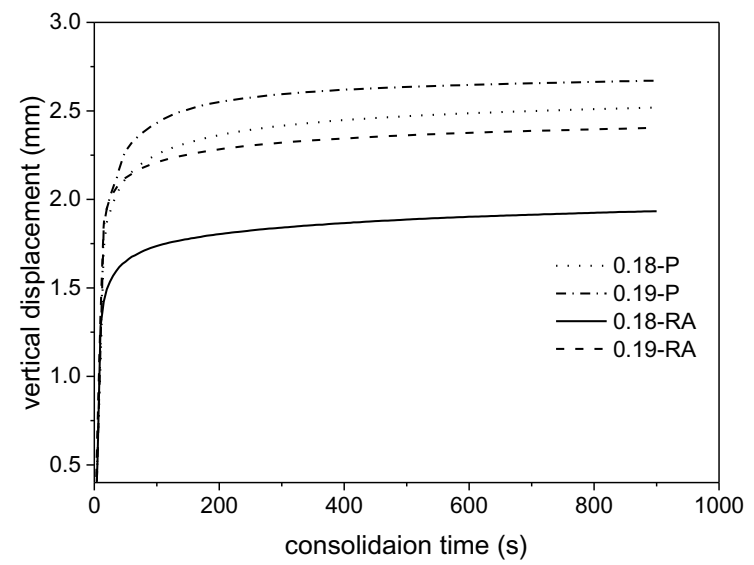

Fig. 2. Maximum shear stress vs normal stress for mix 0.18-P

\section{Conclusions}

- The increase of the water-to-solid ratio for an AAC paste from 0.18 to 0.19 has a marginal impact on the angle of internal friction but does however, reduce the cohesion by $85 \%$.
- The addition of a cellulosic rheology aid does not necessarily increase the "flow-like" characteristics of the mix which provides it plasticity and the ability to move through the extruder.

- Cellulosic rheology aid has the potential to significantly improve the AAC paste 'green strength', minimising the risk of the paste from collapsing after extrusion.

\section{References}

1. Y. Shao, S. P. Shah, and S. Marikunte, Concr. Int., 17, 4 (1995)

2. Y. Shao. and S. P. Shah, Mater. J., 94, 6 (1997)

3. C. Aldea, S. Marikunte, and S. P. Shah, Adv. Cem. Based Mater., 8, 2 (1998)

4. Z. Li, S. N. C. Chui, and M. Bin, Mater. J., 96, 5 (1999)

5. X. Zhou and Z. Li, Constr. Build. Mater., 27, 1 (2012)

6."Green INSTRUCT," 2016. [Online]. Available: http://www.greeninstruct.eu/. [Accessed: 09-Jul-2017].

7. X. Zhou, Z. Li, M. Fan, and H. Chen, Cem. Concr. Compos., 37, 35 (2013)

8. R. Alfani, G. L. Guerrini, Mater. Struct., 38, 2 (2005)

9. A. Cerato and A. Lutenegger, Geotech. Test. J., 29, 6 (2006) 